\title{
Ordering in Blends of Diblock Copolymers
}

\author{
Nagraj Koneripalli, ${ }^{*, \dagger}$ Rastislav Levicky, ${ }^{\ddagger}$ and Frank S. Bates* \\ Department of Chemical Engineering and Materials Science, University of Minnesota, \\ Minneapolis, Minnesota 55455
}

\section{Mark W. Matsen}

Polymer Science Centre, University of Reading, Whiteknights, Reading RG6 6AF, U.K.

\author{
Sushil K. Satija
}

Reactor Radiation Division, National Institute of Standards and Technology, Gaithersburg, Maryland 20899

\section{J ohn Ankner and Helmut Kaiser}

Missouri University Research Reactor, Columbia, Missouri 65211

Received August 14, 1997; Revised Manuscript Received February 26, 1998

\begin{abstract}
The morphology of binary blends of poly(styrene)-poly(2-vinylpyridine) (PS-PVP) diblock copolymers of the type $(A-B)_{\alpha}+(A-B)_{\beta}$ was examined in a thin-film geometry using neutron reflection (NR), transmission electron microscopy (TEM), and self-consistent field (SCF) calculations. Blends of symmetric diblock copolymers $\left(\mathrm{f}_{\mathrm{A}, \alpha} \approx \mathrm{f}_{\mathrm{A}, \beta} \approx 0.5\right)$ revealed a localization of the lower molecular weight diblock copolymer and stretching of the higher molecular weight diblock copolymer near the AB interface that is qualitatively consistent with the data of a prior study by Mayes and co-workers. Blends of asymmetric diblock copolymers of the type $\mathrm{f}_{\mathrm{A}, \alpha}<0.5$ and $\mathrm{f}_{\mathrm{A}, \beta}>0.5$ having similar molecular weights $\left(\mathrm{N}_{\alpha}\right.$ $\approx \mathrm{N}_{\beta}$ ) and with $\sim 50 \%$ overall composition of a chemically similar block in the blend, $\left\langle\mathrm{f}_{\mathrm{A}}\right\rangle \approx 0.5$, revealed molecular-level mixing of the two components and the formation of a single-phase morphology. A lamellar morphology was formed even when the individual components exhibit a nonlamellar morphology in the pure melt state. In general, the morphological characteristics are well anticipated by SCF calculations. After accounting for fluctuations at the interface, spatial distributions of the individual components in the blend obtained by SCF calculations were in close agreement with the NR data. For diblock copolymers near the order-disorder transition, a $\chi$ value higher than the bulk was necessary to fit the experimental data, suggesting an enhancement of ordering in the thin films near the transition due to the presence of the surfaces.
\end{abstract}

\section{Introduction}

Diblock copolymers exhibit a wide array of morphologies in the ordered state ranging from a simple layered morphology to a complex bicontinuous structure. In classical treatments of the thermodynamics of diblock copolymers, the phase behavior is governed by two parameters: (a) $f_{A}$, the volume fraction of the $A$ block in an $\mathrm{A}-\mathrm{B}$ diblock copolymer; and (b) $\chi \mathrm{N}$, the product of the $A B$ interaction parameter $(\chi)$ and the number of repeating units in the polymer chain (N). The symmetry of the microstructure (viz. lamellar, hexagonal, $B C C$, etc.) is chiefly determined by $f_{A}$ and to a smaller extent by $\chi \mathrm{N}$. Nonetheless, in many instances, varying $\mathrm{f}_{\mathrm{A}}$ is the only means of influencing a particular morphology in the diblock copolymer morphology map. This method of controlling the morphology involves the synthesis of copolymers having a particular $f_{A}$ and is tedious and may not be economically viable.

Blending is an attractive alternative to manipulate structural properties of diblock copolymers. Blending offers the potential to access properties intermediate to those of the pure components in a continuous manner.

\footnotetext{
† Present address: 3M Corporate Research Science Research Laboratory, 201-2N-19, 3M Center, St. Paul, MN 55144.

₹ Present address National Institute of Standards and Technology, Gaithersburg, MD 20899.

* Authors for correspondence: <nagraj@mmm.com>, <bates@ cems.umn.edu> .
}

The parameters that dictate the thermodynamics of a blend are the molecular characteristics of the individual components and the blend composition. The number of parameters can easily get large. Even in the simplest system, i.e., binary blends $(\alpha+\beta)$ of $A B$ diblock copolymers, $(A-B)_{\alpha} /(A-B)_{\beta}$, there are six parameters that control the phase behavior of the blend: $A B$ segment-segment interaction parameter $\chi$, degrees of polymerization $\mathrm{N}_{\alpha}$ and $\mathrm{N}_{\beta}$, volume fraction of the $\mathrm{A}$ block in the individual copolymers $\mathrm{f}_{\mathrm{A}, \alpha}$ and $\mathrm{f}_{\mathrm{A}, \beta}$, and volume fraction of the $\alpha$ component in the blend $\left(\phi_{\alpha}\right)$. Experimental ${ }^{1-9}$ investigations have been designed to provide insights into the general structural features of diblock copolymer blends al ong with theoretical efforts ${ }^{10-13}$ that have furnished detailed analyses of the blend-phase behavior. These studies have focused on two issues pertaining to blending: (a) the miscibility of the constituents of the blend and (b) the morphology of the blend in relation to the morphology of the pure diblock copolymer components.

A quantitative comparison of the structural features predicted by the theoretical models to the experimental data in binary blends of symmetric and asymmetric diblock copolymers has not been reported previously, to the best of our knowledge. In this report, we test the predictive power of SCF calculations through comparison with composition profiles in binary blends of diblock copolymers obtained from experiment. Blends of poly- 
Table 1. Molecular Characteristics of the PS-PVP Diblock Copolymers Employed in This Study

\begin{tabular}{|c|c|c|c|c|}
\hline $\begin{array}{c}\text { PS-PVP } \\
\text { copolymers a }\end{array}$ & $\begin{array}{c}10^{-3} \mathrm{M}_{\mathrm{n}} \\
\mathrm{g} / \mathrm{mol}\end{array}$ & $M_{w} / M_{n}$ & $f_{P S}{ }^{b}$ & morphologyc \\
\hline $\begin{array}{l}\text { SV25/d48 (d60) } \\
\text { SV26/d48 (d120) } \\
\text { SV27/h49 (h120) }\end{array}$ & $\begin{array}{r}57 \\
124 \\
110\end{array}$ & $\begin{array}{l}1.09 \\
1.10 \\
1.11\end{array}$ & $\begin{array}{l}0.48 \\
0.48 \\
0.49\end{array}$ & $\begin{array}{l}\text { LAM }^{d} \\
\text { LAM }^{d} \\
\text { LAM }^{d}\end{array}$ \\
\hline $\begin{array}{l}\text { SV4/h30 } \\
\text { SV10/d35 } \\
\text { SV8/d38 } \\
\text { SV12/d40 } \\
\text { SV13/d63 } \\
\text { SV9/d65 } \\
\text { SV1/h68 } \\
\text { SV14/d70 }\end{array}$ & $\begin{array}{l}22.2 \\
21.2 \\
20.2 \\
21.8 \\
21.7 \\
20.5 \\
18.7 \\
21.8\end{array}$ & $\begin{array}{l}1.05 \\
1.05 \\
1.05 \\
1.06 \\
1.05 \\
1.05 \\
1.13 \\
1.05\end{array}$ & $\begin{array}{l}0.30_{3} \\
0.34_{8} \\
0.37_{5} \\
0.39_{9} \\
0.63_{2} \\
0.65_{2} \\
0.67_{9} \\
0.70_{3}\end{array}$ & $\begin{array}{l}\text { HEXe } \\
\text { HEX } \\
\text { la3de } \\
\text { LAM }^{\text {d,e }} \\
\text { LAMe }^{\text {LAM }} \\
\text { HPL e }\end{array}$ \\
\hline
\end{tabular}

a dPS and hPS refer to perdeuterated and unlabeled poly(styrene) blocks in the copolymer. Details of the synthesis for the copolymers are provided in ref 14 . ${ }^{\mathrm{b}}$ Calculated using $\rho_{\mathrm{hPS}}=1.05$ $\mathrm{g} / \mathrm{cm}^{3}, \rho_{\mathrm{dPS}}=1.13 \mathrm{~g} / \mathrm{cm}^{3}$, and $\rho_{\mathrm{PVP}}=1.14 \mathrm{~g} / \mathrm{cm}^{3}$. ${ }^{\mathrm{C}} \mathrm{LAM}, \mathrm{HEX}, \mathrm{HPL}$, and la3d notation correspond to lamellar, hexagonal cylinders, hexagonal perforated layered, and gyroid morphologies, respectively. ${ }^{d}$ Morphology of copolymers deduced from reflectivity measurements this work and ref 28. e Morphology of copolymers deduced from SANS, TEM, and rheology. ${ }^{14}$

(styrene)-poly(2-vinylpyridine) (PS-PVP) diblock copolymers were investigated in two sets of controlled experiments. In the first set of experiments, we examined the morphology of a binary blend of symmetric diblock copolymers with varying fractions of one of the diblocks (varying $\phi_{\alpha}$, constant $\mathrm{f}_{\mathrm{A}, \alpha} \approx \mathrm{f}_{\mathrm{A}, \beta} \approx 0.5$, and constant $\mathrm{N}_{\alpha} / \mathrm{N}_{\beta}$ ). In the second set of experiments, we studied the morphology of binary blends of asymmetric diblock copolymers, with $\mathrm{f}_{\mathrm{A}, \alpha}+\mathrm{f}_{\mathrm{A}, \beta} \approx 1$ and $\mathrm{N}_{\alpha} \approx \mathrm{N}_{\beta}$ and varying $\Delta f=\left|f_{A, \alpha}-f_{A, \beta}\right|$. Neutron reflectivity (NR) and transmission electron microscopy (TE M) were employed as complementary methods to examine the morphol ogy in the direction normal to the substrate (NR) and in the plane containing the substrate/film interface (TEM). NR measurements provide quantitative information on the blend morphology. PS composition profiles in the blends were obtained by deuterium labeling the PS block in one of the components. The volume fraction profiles obtained from NR were compared with the SCF calculations.

\section{Experimental Section}

PS-PVP diblock copolymers with both hydrogenated (hPS) and perdeuterated (dPS) polystyrene block were employed in the experiments. The copolymers were synthesized by one of us, Rastislav Levicky (symmetric copolymers), and by Mark F. Schulz (asymmetric copolymers) using the sequential anionic polymerization technique described elsewhere. ${ }^{14}$ The molecular characteristics of the copolymers used in this study are presented in Table 1. Copolymers in this report are referenced by the abbreviation indicated in the first column of Table 1. Copolymers in the first three rows of the table are symmetric diblock copolymers, while the rest are asymmetric diblock copolymers.

Thin films of copolymer blends for reflection measurements were prepared in a class 10 clean room. Silicon substrates of diameter 10 or $5 \mathrm{~cm}$ having a nominal thickness of $0.476 \mathrm{~cm}$ and (111) orientation were used in this study. Substrates were cleaned in a 30-70\% hydrogen peroxide-sulfuric acid mixture at $120^{\circ} \mathrm{C}$ for $5 \mathrm{~min}$, rinsed in deionized water, and dried with ultrahigh-purity nitrogen. Prior to spin coating, thethickness of native oxide (generally 10-25 $\AA$ ) on the substrate was measured by ellipsometry. Solutions for spin-coating thin films of copolymer blends were prepared by co-dissolving measured quantities of two copolymers in toluene foll owed by filtering using a $0.45-\mu \mathrm{m}$ PTFE filter. A few milliliters of the filtered solution was placed on the cleaned substrate and spun at speeds ranging from 1000 to $2500 \mathrm{rpm}$. Spinning speeds and the concentration of the copolymer solution were appropriately changed to obtain films of the desired thickness.

The copolymer films were annealed for $72 \mathrm{~h}$ at $165-170^{\circ} \mathrm{C}$ in a vacuum ( $20 \mathrm{mTorr}$ ). Prior to annealing, the film thickness was measured by ellipsometry and X-ray reflectivity. In ellipsometric measurements of PS-PVP films, the refractive index for $\lambda=6328 \AA$ was fixed at $1.58 .{ }^{15} \mathrm{X}$-ray measurements of the film thickness were facilitated by the large electron density contrast between the copolymer film and the substrate and the weak electron density contrast between PS and PVP which resulted in well-resolved oscillations in the data. The frequency of these oscillations was used to determine the film thickness. Film thickness measurements by these two techniques are related to each other through the oxide layer thickness. Due to a large electron density contrast between the copolymer film and the native oxide layer, the thickness measurement by X-ray reflectivity yields the copolymer film thickness, while owing to a weak refractive index contrast between the copolymer film and the native oxide, ellipsometric measurements yield the sum of the native oxide and the copolymer film thickness. After correcting for the native oxide thickness, the agreement between the two methods was better than $20 \AA$.

Wetting of the substrate by the PVP block and segregation of the dPS block to the air interface due to a lower surface tension induces an orientation in the film morphology. In symmetric diblock copolymers, this results in lamellae oriented parallel to the two surfaces. If the film thickness is not compatible with the domain period in the film, the bilayer adjacent to the free surface (air) is incomplete with regions of islands or holes. ${ }^{16}$ These features on the free surface complicate NR data analysis, particularly in very thin films. To facilitate NR data analysis, the presence of these features in annealed films was minimized by appropriately adjusting the amount of spin-coated copolymer. Several attempts were often necessary to generate optimum samples with minimum $(<15 \%)$ islands/holes.

The composition of a spin-coated film was evaluated by gel permeation chromatography (GPC) and compared with the values used to prepare spin-coating solutions. This was done to assess if the procedures employed for spin coating (e.g., filtration) altered the composition of the blend in the thin film. These tests were performed on binary mixtures of $d 60 / h 120$ (see Table 1) on two test samples having different blend compositions. Thethin-film blend composition was determined by linearly combining the pure component GPC traces to fit the GPC trace of the blend. The compositions determined from the GPC analysis of thin films and the original spin-coating solutions were within $4 \%$.

Neutron reflection measurements were conducted at the National Institute of Standards and Technology (NIST, Gaithersburg, $M D)^{17}$ and at the Missouri University Research Reactor (MURR, Columbia, MO) ${ }^{18}$ on fixed-wavelength reflectometers. The wavelengths of the radiation used were $4.77 \AA$ (NIST) and $2.35 \AA$ (MURR). The resolution of the instruments $(\Delta \mathrm{k} / \mathrm{k})$ under the conditions used to collimate the neutron beam was determined by fitting the reflectivity data from a standard sample and was found to be 0.027 (half-width at halfmaximum of the Gaussian spread). Background signals were measured on select samples with the detector offset by $\sim 0.1^{\circ}$ from specular reflectivity. This correction was insignificant for the experiments described here. The coherent scattering length densities (SLDs) of dPS, hPS, PVP, $\mathrm{SiO}_{x}$ and $\mathrm{Si}$ (substrate) were calculated as $6.42 \times 10^{-6}, 1.43 \times 10^{-6}, 1.93$ $\times 10^{-6}, 3.45 \times 10^{-6}$, and $2.08 \times 10^{-6}$, respectively, in units of $\AA^{-2}$. Correction due to incoherent scattering and absorption was insignificant. Neutron reflectivity data were analyzed by constructing layers of constant SLDs and smearing the interfaces with an error function. Reflectivity curves were calculated from the assumed SLD profiles and compared with measured data. This procedure was carried out iteratively by empl oying statistical error minimization techniques until the agreement between the calculated reflectivity and the experimental reflectivity data was satisfactory. 
In thin films of copolymers having in-plane structure, morphology characterization by neutron reflectivity was not straightforward. Hence, transmission electron microscopy (TEM) was used to characterize the lateral structure in the film. Thin films of the copolymer were spun-cast from filtered toluene solutions on approximately 4-cm-diameter mica substrates. Film thicknesses were measured by X-ray reflectivity, and the samples were thermally annealed. Films were floated off the substrate onto deionized water and captured on 400mesh $\mathrm{Cu}$ grids and then stained in $\mathrm{I}_{2}$ vapor for $\sim 6 \mathrm{~h}$ and examined using a J EOL 1210 instrument operating at $120 \mathrm{kV}$.

\section{Theory}

Our experimental results are compared to the SCF theory of Helfand. ${ }^{19}$ The extension of this theory to multicomponent blends is described for the canonical ensemble by Hong and Noolandi 20 and for the grandcanonical ensemble by Matsen. ${ }^{21}$ The latter ensemble, in which concentrations are controlled by a chemical potential, is most appropriate for calculating phase boundaries and coexistence regions. Our calculations focus on the one-phase regions with a fixed concentration $\phi_{\alpha}$, so we employ the canonical ensemble.

In the self-consistent theory, the molecular interactions experienced by the $A$ and $B$ segments are replaced by the fields, $w_{A}(r)$ and $w_{B}(r)$, respectively. This allows the statistical mechanics of each $A B$ diblock copolymer species to be evaluated. To do this, the contour of the $(A B)_{\alpha}$ diblock is parametrized from its $A$ end to its $B$ end by $s=0$ to $N_{\alpha}$. Then the partition function, $q_{\alpha^{-}}$ $(\mathbf{r}, \mathbf{s})$, of the $(0, s)$ portion of the chain is calculated with segment $s$ fixed at $\mathbf{r}$. It satisfies

$$
\begin{aligned}
& \frac{\partial}{\partial \mathrm{s}} \mathrm{q}_{\alpha}(\mathbf{r}, \mathrm{s}) \\
& -\left\{\begin{array}{l}
\frac{1}{6} \mathrm{a}_{\mathrm{A}}^{2} \nabla^{2} \mathrm{q}_{\alpha}(\mathbf{r}, \mathrm{s})-\mathrm{w}_{\mathrm{A}}(\mathbf{r}) \mathrm{q}_{\alpha}(\mathbf{r}, \mathrm{s}) \text { if } 0<\mathrm{s}<\mathrm{f}_{\mathrm{A}, \alpha} \mathrm{N}_{\alpha} \\
\frac{1}{6} \mathrm{a}_{\mathrm{B}}^{2} \nabla^{2} \mathrm{q}_{\alpha}(\mathbf{r}, \mathrm{s})-\mathrm{w}_{\mathrm{B}}(\mathbf{r}) \mathrm{q}_{\alpha}(\mathbf{r}, \mathrm{s}) \text { if } \mathrm{f}_{\mathrm{A}, \alpha} \mathrm{N}_{\alpha}<\mathrm{s}<\mathrm{N}_{\alpha}
\end{array}\right.
\end{aligned}
$$

with the initial condition $q_{\alpha}(\mathbf{r}, 0)=1$. For the diblock molecules examined here, we assume that the $A$ and $B$ statistical segment lengths, $a_{A}$ and $a_{B}$, are equal. Next, a second partition function, $q_{\alpha}^{*}(\mathbf{r}, \mathrm{s})$, is calculated for the $\left(\mathrm{s}, \mathrm{N}_{\alpha}\right)$ portion of the chain with segment $\mathrm{s}$ fixed at $\mathbf{r}$. It satisfies eq 1 with the right-hand side multiplied by -1 and the initial condition $\mathrm{q}^{*}\left(\mathbf{r}, \mathrm{N}_{\alpha}\right)=1$. The total partition function of the molecule is

$$
\mathrm{Q}_{\alpha}=\int \mathrm{q}_{\alpha}(\mathbf{r}, \mathrm{s}) \mathrm{q}_{\alpha}^{*}(\mathbf{r}, \mathrm{s}) \mathrm{dr}
$$

which can be evaluated with any value of s. Once this procedure is repeated for the $(A B)_{\beta}$ diblock molecule, the $A$ and $B$ segment distributions can be calculated using

$$
\begin{gathered}
\phi_{\mathrm{A}}(\mathbf{r})=\frac{\mathrm{V} \phi_{\alpha}}{\mathrm{Q}_{\alpha} \mathrm{N}_{\alpha}}\left(\int_{0}^{\mathrm{f}_{\mathrm{A}, \alpha} \mathrm{N}_{\alpha}} \mathrm{q}_{\alpha}(\mathbf{r}, \mathbf{s}) \mathrm{q}_{\alpha}^{*}(\mathbf{r}, \mathbf{s}) \mathrm{ds}\right)+ \\
\frac{\mathrm{V}\left(1-\phi_{\alpha}\right)}{\mathrm{Q}_{\beta} \mathrm{N}_{\beta}}\left(\int_{0}^{\mathrm{f}_{\mathrm{A}, \beta} \mathrm{N}_{\beta}} \mathrm{q}_{\beta}(\mathbf{r}, \mathbf{s}) \mathrm{q}_{\beta}^{*}(\mathbf{r}, \mathrm{s}) \mathrm{ds}\right) \\
\phi_{\mathrm{B}}(\mathbf{r})=\frac{\mathrm{V} \phi_{\alpha}}{\mathrm{Q}_{\alpha} \mathrm{N}_{\alpha}}\left(\int_{\mathrm{f}_{\mathrm{A}, \alpha} \mathrm{N}_{\alpha}}^{\mathrm{N}_{\alpha}} \mathrm{q}_{\alpha}(\mathbf{r}, \mathbf{s}) \mathrm{q}_{\alpha}^{*}(\mathbf{r}, \mathrm{s}) \mathrm{ds}\right)+ \\
\frac{\mathrm{V}\left(1-\phi_{\alpha}\right)}{\mathrm{Q}_{\beta} \mathrm{N}_{\beta}}\left(\int_{\mathrm{f}_{\mathrm{A}, \beta} \mathrm{N}_{\beta}}^{\mathrm{N}_{\beta}} \mathrm{q}_{\beta}(\mathbf{r}, \mathbf{s}) \mathrm{q}_{\beta}^{*}(\mathbf{r}, \mathbf{s}) \mathrm{ds}\right)
\end{gathered}
$$

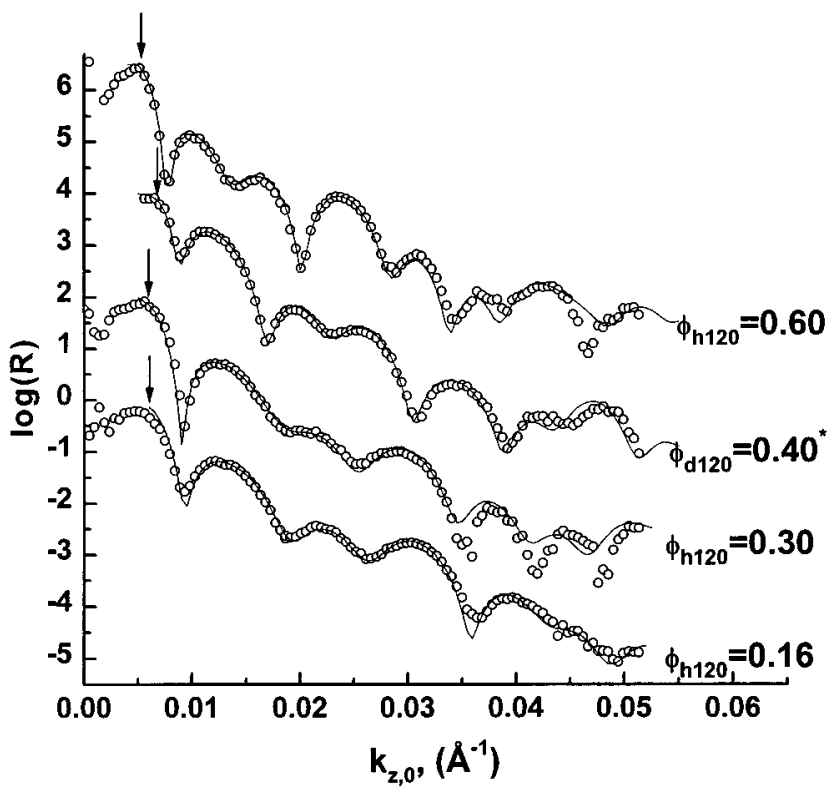

Figure 1. Neutron reflectivity data (open symbols) and the calculated reflectivity (solid lines) for a binary blend of h120/ d60 PS-PVP diblock copolymers with $\phi_{\mathrm{h} 120}=0.16,0.30$, and 0.60 and a blend of $\mathrm{d} 120 / \mathrm{d} 60$ with $\phi_{\mathrm{d} 120}=0.40$. The sample with $\phi_{\mathrm{d} 120}=0.40$ is a blend of $\mathrm{d} 120 / \mathrm{d} 60$ (the PS block in both components is perdeuterated), while the rest of the samples, $\phi_{\mathrm{h} 120}=0.16,0.30$, and 0.60 , are blends of h120/d60 (the PS block of the shorter diblock is perdeuterated). The arrows indicate the critical wave vector, $\mathrm{k}_{\mathrm{c}}$ in the profiles.

In each expression, the two terms correspond to contributions from the $(A B)_{\alpha}$ and $(A B)_{\beta}$ molecules, respectively.

The fields representing the molecular interactions must be adjusted to satisfy the self-consistent field equations, $\mathrm{w}_{\mathrm{A}}(\mathbf{r})=\chi \phi_{\mathrm{B}}(\mathbf{r})+\xi(\mathbf{r})$ and $\mathrm{w}_{\mathrm{B}}(\mathbf{r})=\chi \phi_{\mathrm{A}}(\mathbf{r})+$ $\xi(\mathbf{r})$. The function $\xi(\mathbf{r})$ is a Lagrange multiplier used to enforce the constraint of bulk incompressibility, $\phi_{A}(\mathbf{r})$ $+\phi_{\mathrm{B}}(\mathbf{r})=1$. The average value of $\xi(\mathbf{r})$ is immaterial, so we set $\int \xi(\mathbf{r}) \mathrm{d} \mathbf{r}=0$. In the present study, we numerically solve these equations for the lamellar phase where all the functions are uniform in the $x$ and $y$ directions and periodic in the $z$ direction with a period D. Once the solution is obtained, the free energy of the lamellar phase is given by

$$
\begin{array}{r}
\frac{\mathrm{F}}{\mathrm{k}_{\mathrm{B}} T \rho_{\rho_{0}}}=-\frac{\phi_{\alpha}}{\mathrm{N}_{\alpha}} \ln \mathrm{Q}_{\alpha}-\frac{1-\phi_{\alpha}}{\mathrm{N}_{\beta}} \ln \mathrm{Q}_{\beta}+ \\
\chi \int \phi_{\mathrm{A}}(\mathbf{r}) \phi_{\mathrm{B}}(\mathbf{r}) \mathrm{d} \mathbf{r}
\end{array}
$$

where $\mathrm{V}$ is the volume of the melt and $\rho_{0}$ is a common segment density. The final step is to minimize $F$ with respect to $D$ in order to find the equilibrium domain spacing.

\section{Results and Discussion}

Blends of Symmetric Diblock Copolymers. Figure 1 shows the neutron reflectivity profiles from binary blends of PS-PVP diblock copolymers. The molecular characteristics of these copolymers are given in the first three rows of Table 1 . The copolymers will be referenced as d60, d120, and h120, where the letter of the alphabet indicates whether the PS block is protonated or perdeuterated and the number is the approximate molecular weight in thousands. Data from a h120/d60 blend having volume fractions of h120 equal to 0.16 , 
0.30 , and 0.60 and a blend of $\mathrm{d} 120 / \mathrm{d} 60$ having a volume fraction of d120 equal to 0.40 are presented in Figure 1 . In the samples with $\phi_{\mathrm{h} 120}=0.16,0.30$, and 0.60 , only the PS block of the smaller molecular weight component (d60) is perdeuterated, whereas in the sample with $\phi_{\mathrm{d} 120}$ $=0.40$, the PS blocks of both components are perdeuterated. The calculated reflectivities are shown as solid lines in Figure 1. At low values of $k_{z, 0}$, departure in the reflectivity data from the idealized total reflection $(\log (R)=0)$ is an experimental artifact. This occurs because the projected area of the neutron beam at low angles is greater than the area of the sample. Data with $\mathrm{k}_{z, 0}$ smaller than $0.006 \AA^{-1}$ were not used in the reflectivity calculations. The position of the critical wave vector, $k_{c}$, are indicated by arrows. This value varies in correspondence with the average SLDs of the film (or, in this case, the blend composition).

The SLD profiles used to calculate the reflectivities in Figure 1 are shown in Figure 2. In these profiles, the film/air interface occurs at $z=50 \AA$. The sharp spike in the profiles at higher values of $z$ is due to the thin native oxide $(\sim 15 \AA)$ present on $\mathrm{Si}$ substrates. This feature is representative of the film/substrate interface in all the SLD profiles. The PS-rich and the PVP-rich regions are labeled in the top SLD profile and can be similarly identified in the other SLD profiles. All the samples composed of 1.5 bilayers with PS and PVP blocks located at the air and substrate interfaces, respectively. In profiles with $\phi_{\mathrm{h} 120}=0.16,0.30$, and 0.60 , nonuniformity of the SLD in the PS domain occurs due to the difference in the SLD values of the dPS and hPS chains (higher and lower SLDs, respectively). The profiles show a local ization of the dPS (short) chains to the PS/PVP interface and hPS (long) chains to the center of the PS domain. The degree of nonuniformity in the SLD of the PS region increases as the volume fraction of h120 increases in the blend (see profiles with $\phi_{\mathrm{h} 120}=$ $0.16,0.30$, and 0.60 ). In the $\phi_{\mathrm{h} 120}=0.60$ sample, however, there is considerable asymmetry in the distribution of the short and the long PS chains in the domain adjacent to the substrate. In the monolayer immediately adjacent to the substrate, there is a greater fraction of the short chains than the film average. Such inhomogeneities in the distribution of the components could be due to strong adsorption of the PVP to the substrate that would necessitate considerably longer annealing periods to eliminate asymmetric chain distributions. The asymmetry in the chain distributions present in the $\phi_{\text {h120 }}=0.60$ sample and not present in the other samples suggests that this is a nonequilibrium-type effect. The average calculated SLD of the film in all the profiles was within 3\% of the experimental composition. In the sample, with $\phi_{\mathrm{d} 120}=0.40$, both the short and the long PS chains are deuterated and, hence, the PS regions appear uniform. The slight decrease in the SLD in the PS domain adjacent to the air interface is due to the presence of holes on the free surface.

The localization of short and long chains within a domain of a binary blend of diblocks has been predicted theoretically ${ }^{12,13}$ and observed experimentally. ${ }^{7}$ To compare our experimental findings with the predicted profiles from self-consistent field calculations, the SLD profiles were transformed to vol ume fraction profiles as follows. First, the SLD profile from the $\mathrm{d} 120 / \mathrm{d} 60$ blend $\left(\phi_{\mathrm{d} 120}=0.40\right)$ was inverted to volume fraction profiles using the known SLD values of dPS and PVP and the measured interfacial profile between the dPS and the

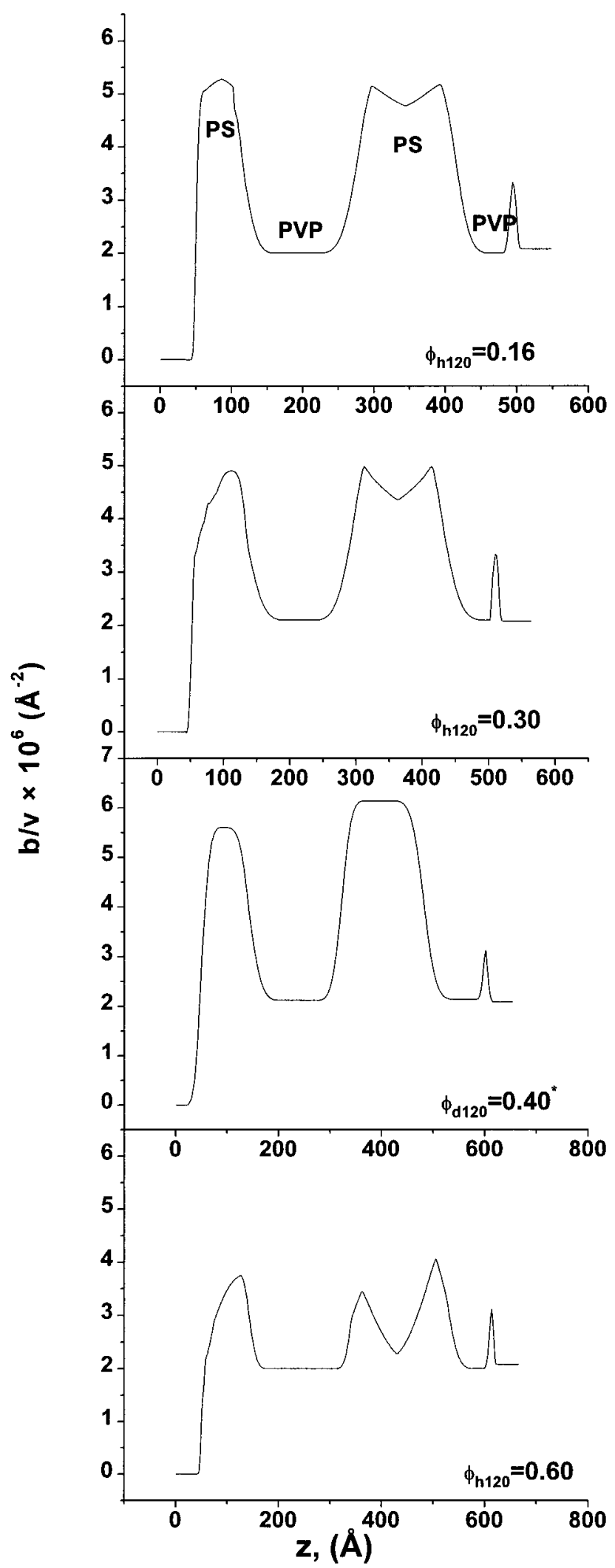

Figure 2. SLD profiles used to calculate the reflectivities shown in Figure 1. The film/air interface in all the profiles is at $z=50 \AA$. The spike in the SLD profile at approximately $z$ $=600 \AA$ is the native oxide and denotes the film/substrate interface.

PVP domains. Next, it was assumed that in blends of h120/d60, the interfacial region between the PS and PVP was described by the profile obtained from the d120/d60 sample. From the SLD profile of the h120/ 
d60 blend and known SLD values for hPS, dPS, and PVP, the volume fraction profiles of the hPS and dPS blocks were calculated. The assumptions associated with such calculations are (i) there is a negligible isotopic effect between dPS and hPS and (ii) the interfacial profile between the PS and PVP domains is not affected by the blend composition. The latter is shown to be a good assumption from the results presented below.

Volume fraction profiles of the dPS block in blends of h120/d60 and profiles from SCF calculations are compared in Figure 3. The profiles are from the bilayer adjacent to the substrate. The bilayer dimensions are normalized so that it extends from arbitrary units of -0.5 to +0.5 . The molecular characteristics of the copolymers, viz. $\mathrm{f}_{\mathrm{A}, \alpha}, \mathrm{f}_{\mathrm{A}, \beta}, \mathrm{N}_{\alpha}$, and $\mathrm{N}_{\beta}$, used for SCF calculations are listed in Table 1 . In the absence of experimental data, the statistical segment length of PVP was assumed to be identical to that of (structurally similar) PS. Accurate data on the Flory-Huggins interaction parameter $(\chi)$ are difficult to obtain. Indirect methods, such as fitting experimental data to theory, are generally used to estimate $\chi$. For PS and PVP, the $\chi$ parameter at $170{ }^{\circ} \mathrm{C}$ is estimated to be $\approx 0.1 .^{22,23} \mathrm{~F}$ or purposes of comparison, SCF calculated profiles for $\chi=$ 0.10 as well as $\chi=0.14$ are plotted together with the experimental data. The agreement between the experimental data and the SCF profiles is reasonably good for the samples with $\phi_{\mathrm{h} 120}=0.16$ and 0.30. In the strongly segregated regime, the SCF profiles for $\chi=0.10$ and 0.14 are not very different from one another, although it appears that the $\chi=0.10$ profiles are more closely matched with the experimental data. The interfacial width between PS and PVP domains from the SCF calculations is sharper than suggested by experiment. This discrepancy is discussed below. In the sample with $\phi_{\mathrm{h} 120}=0.60$, however, asymmetry in the chain distributions precludes a direct comparison with the SCF calculations. From the SLD profile for the $\phi_{\mathrm{h} 120}=0.60$ sample in Figure 2 , it is clear that there is a greater concentration of d60 chains adjacent to the substrate and a depletion of d60 chains in the center domain. Thus, the PVP domain width adjacent to the substrate is proportionately smaller than the results in the artificial spike (due to the native oxide) in the composition profile in Figure 3 . The qualitative features of the profile, such as the volume fraction of dPS at the center of the domain, are in agreement with the SCF calculations. The domain spacings were also in good quantitative agreement with SCF calculations, as well as with the SSL result derived in (12) and other diblock copolymer systems. ${ }^{1,7}$

Blends of Asymmetric Diblock Copolymers. The molecular characteristics of the asymmetric diblock copolymers used in this study are given in the bottom half of Table 1. The copolymers are identified by the abbreviations indicated in the table. For example, in the sample SV4/h30, "SV4" refers to the sequence number of the synthesized PS-PVP diblock copolymer (consistent with ref 14) and "h30" refers to a hydrogenated PS block in the diblock copolymer with volume fraction of $30 \%$.

Figure 4 shows the reflectivity profiles from the neat diblock copolymer samples SV12/d40 and SV10/d35. The reflectivity data from SV12/d40 were analyzed employing methods described previously, and the SLD profile obtained is presented in Figure 5. The copolymer

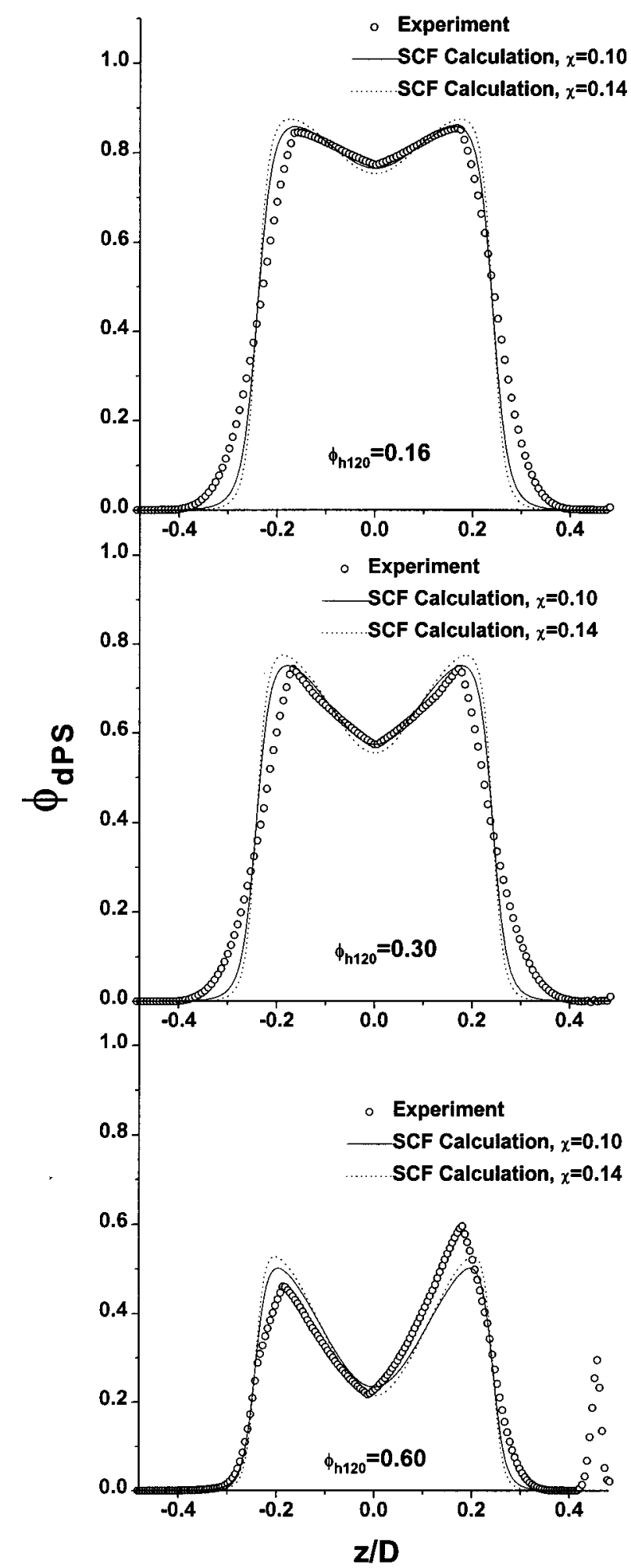

Figure 3. Volume fraction of dPS (short) chains from experiment (open symbols) and self-consistent field calculations (solid lines) for three blend compositions. Profiles correspond to the samples of Figure 1 and Figure 2.

sample SV12/d40 exhibits a lamellar morphology with $\mathrm{dPS}$ and PVP domains parallel to the substrate and the dPS block segregating to the air interface while the PVP block is segregated to the substrate interface. The small decrease in the SLD values in the profile adjacent to the air interface is due to the presence of holes on the free surface of the film. The width of a dPS domain is about $40 \%$ of the spatial periodicity of the film, consis- 


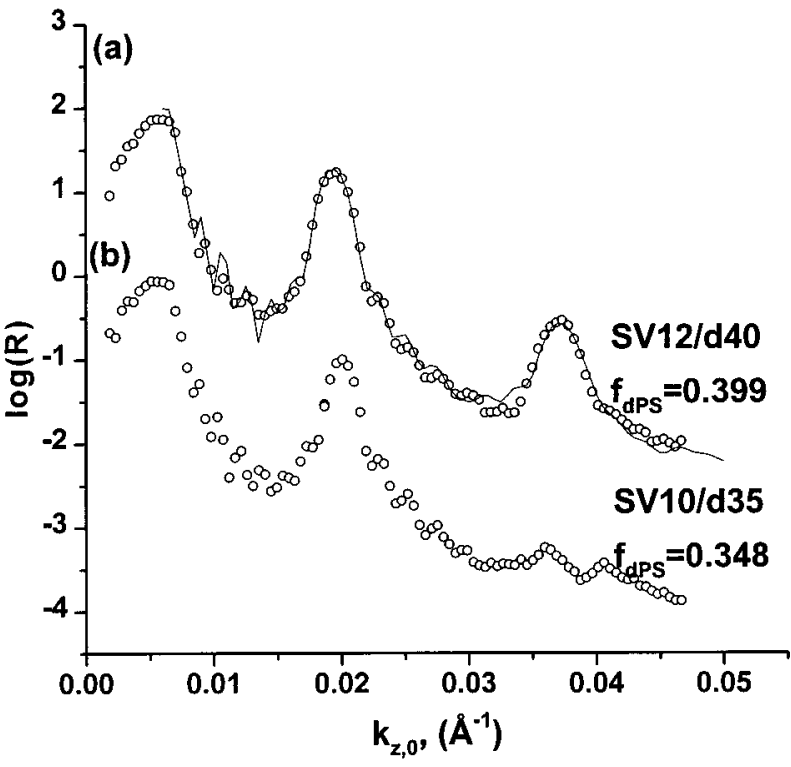

Figure 4. Neutron reflectivity data (open symbols) from samples SV12/d40 (a) and SV10/d35 (b). The solid line is the calculated reflectivity profile from an assumed SLD profile shown in Figure 5. Neutron reflection fit for sample b was not obtained. The in-plane structure for sample b is shown in Figure 6.

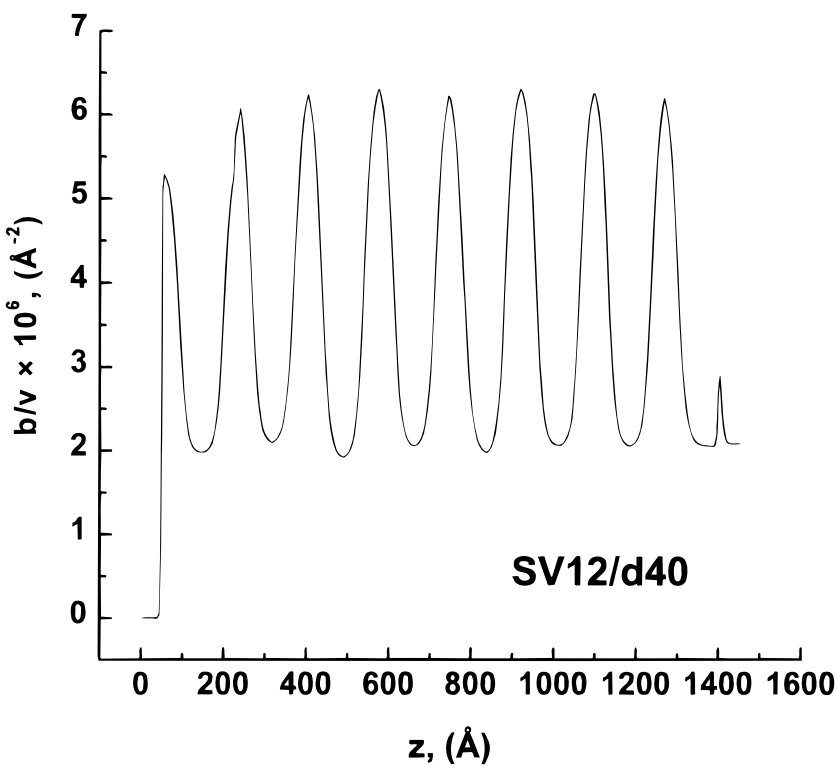

Figure 5. Scattering length density (SLD) profile in sample SV12/d40 used to calculate the reflectivity shown in Figure $4 a$.

tent with the $40 \% \mathrm{dPS}$ volume fraction in the copolymer. In general, the presence or absence of Bragg reflections in the reflectivity profiles can be used to make qualitative inferences on the structure in the film. The unequal widths of the domains give rise to strong first- and second-order Bragg reflections in the reflectivity profile (Figure 4a) at $\mathrm{k}_{\mathrm{z}, 0} \sim 0.0195$ and at $\mathrm{k}_{\mathrm{z}, 0} \sim 0.037$, respectively. For alternating domains of equal widths, only odd Bragg reflections $(1,3,5, \ldots)$ would be observed. The presence of even reflections is thus indicative of asymmetry in the domain widths. Sample SV10/d35, which forms a cylindrical morphology in the bulk state, ${ }^{14}$ shows first-order Bragg reflection and only a weak second-order Bragg reflection (Figure $4 \mathrm{~b}$ ). This suggests weak compositional order in the direction perpendicular to the film/substrateinterface. Figure 6 is a TEM image

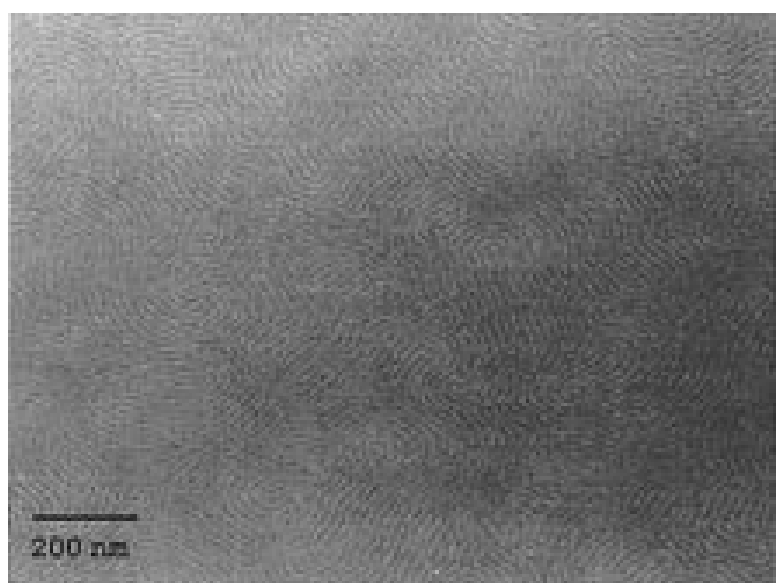

Figure 6. TEM image of a 569- $\AA$-thick film of SV10/d35 spuncast on a mica substrate and annealed at $150{ }^{\circ} \mathrm{C}$ for $72 \mathrm{~h}$. The view direction is perpendicular to the substrate/film interface. The dark regions are iodine-stained PVP domains, while the light regions are the PS domains. The phase-separated morphology is cylinders, and the cylindrical domains are oriented parallel to the film/substrate interface.

that reveals the in-plane structure of the SV10/d35 sample. The film was spun-cast on a mica substrate with a thickness of $569 \AA$ and, then annealed at $150^{\circ} \mathrm{C}$ for $72 \mathrm{~h}$ and imaged in the direction normal to the film/ substrate interface. The image shows lateral correlation with alternating regions of PS (light regions) and iodinestained PVP (dark regions). The bulk morphology of SV10/d35 is cylinders, and the alternating light and dark regions in Figure 6 are indicative of cylindrical domains oriented paralle to the film/substrate interface. A similar TEM measurement on a SV12/d40 sample (not shown) produced an uniform image with no contrast between the PS and PVP domains. This is because the morphology in this sample is lamellar with the lamellar domains oriented with interfaces parallel to the substrate/ film interface (consistent with the NR measurements).

Figure 7 shows the reflectivity profiles obtained from binary blends of asymmetric diblock copolymers. In all these samples, both components of the blend have a perdeuterated poly(styrene) block (dPS) and the overall volume fraction of dPS is about 0.50 . In the blend SV12/ $\mathrm{d} 40+\mathrm{SV} 13 / \mathrm{d} 63$ (Figure 7a), the individual components form a lamellar morphology in the bulk state (see Table 1). As discussed earlier, the neat diblock copolymer, SV12/d40, forms a lamellar morphology with alternating domains of dPS and PVP of unequal widths. The signature of such a morphology is $n$th order $(n=1,2$, $3, \ldots)$ Bragg reflections as revealed by the reflectivity profile in Figure 4a. On blending SV12/d40 and SV13/ d63, however, only the odd-order Bragg reflections ( $\mathrm{n}$ $=1,3, \ldots)$ are dominant and the second-order peak is almost extinct. This suggests nearly equal widths of the dPS and PVP domains and molecular mixing of the two components. This is corroborated by the SLD profile (F igure 8a) used to cal culate the reflectivity curve (Figure 7a). In the binary blend of SV10/d35 + SV9/ d65 (Figure 7b), both pure components exhibit a nonlamellar morphology, and in the blend SV8/d38 + SV13/ d63 (Figure 7c), one of the components (SV8/d38) has a nonlamellar morphology (see Table 1). The blend morphology in both cases, however, is lamellar with dPS and PVP domains of nearly equal widths and the lamellar orientation parallel to the substrate. This can be qualitatively inferred from the reflectivity profiles shown in Figure 7 that reveal dominant odd-order Bragg 


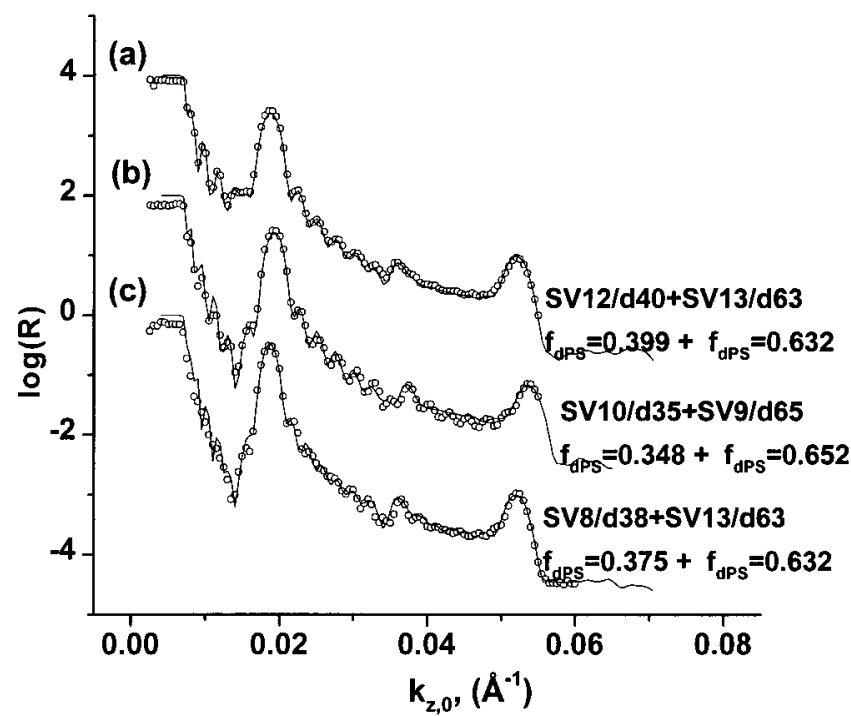

Figure 7. Neutron reflectivity data (open symbols) from binary blends of asymmetric diblock copolymers of dPS-PVP. In all cases, both components of the blend have a perdeuterated poly(styrene) block (dPS). The overall volume fraction of the dPS block in each of the blends is nearly 0.5. The volume fractions of the dPS block in each of the blend components is indicated (see also Table 1). The solid lines are calculated reflectivities with the SLD profiles shown in Figure 8.

reflections and weak even-order reflections and quantitatively from the SLD profiles in Figure 8. All the data in Figure 7 were obtained from approximately $\sim 1200-\AA$ (seven bilayer) films. These relatively large thicknesses were employed so as to minimize the interference between oscillations due to the domains and those from the film thickness. A lamellar morphology is formed in these blends even if the films have a thickness of 450 $\AA$, or $\sim 2.5$ bilayers. This can be inferred from the superposed reflectivity profiles in Figure 9. The two pure components of the blend, SV12/d40 + SV13/d63, form a lamellar morphology; thus, the blend morphology is expected to be lamellar as well. In the blend SV10/ d35 + SV9/d65, even though the pure components do not exhi bit a lamellar structure, the superposition of the reflectivity profile with that of SV12/d40 + SV13/d63 (Figure 9) suggests a lamellar morphology. This is consistent with the data from the thicker films.

The domain period of copolymer SV12/d40 was determined from neutron reflection measurements to be $171 \AA$ (see Figure 4a and Figure 5), while the domain period of the blend SV12/d40 + SV13/d63 was $181 \AA$. This represents a stretching of the domain period by $5.8 \%$ with respect to the pure state. ${ }^{24}$ SCF calculations also predict an increase in domain spacing, but it is somewhat smaller. For $\chi=0.10$ and 0.14 , we calculate a $2.4 \%$ and a $1.7 \%$ increase in the domain size, respectively. The explanation is that a bimodal distribution of blocks can fill space more efficiently than a monodisperse distribution by using its long blocks to fill the center of the domain. Consequently, the entropic stretching energy in the blend is reduced relative to that in the neat diblock melt, which permits the domain size to swell.

In blends of asymmetric diblock copolymers, the difference in the pure component volume fractions ( $\mid \mathrm{f}_{\mathrm{A}, \alpha}$ $\left.-f_{A, \beta} \mid\right)$ is correlated with the degree of asymmetry in the spontaneous curvature of the system. As $\left|f_{A, \alpha}-f_{A, \beta}\right|$ increases, a blend becomes progressively less stable toward miscibility. The $\left|f_{A, \alpha}-f_{A, \beta}\right|$ values for the data

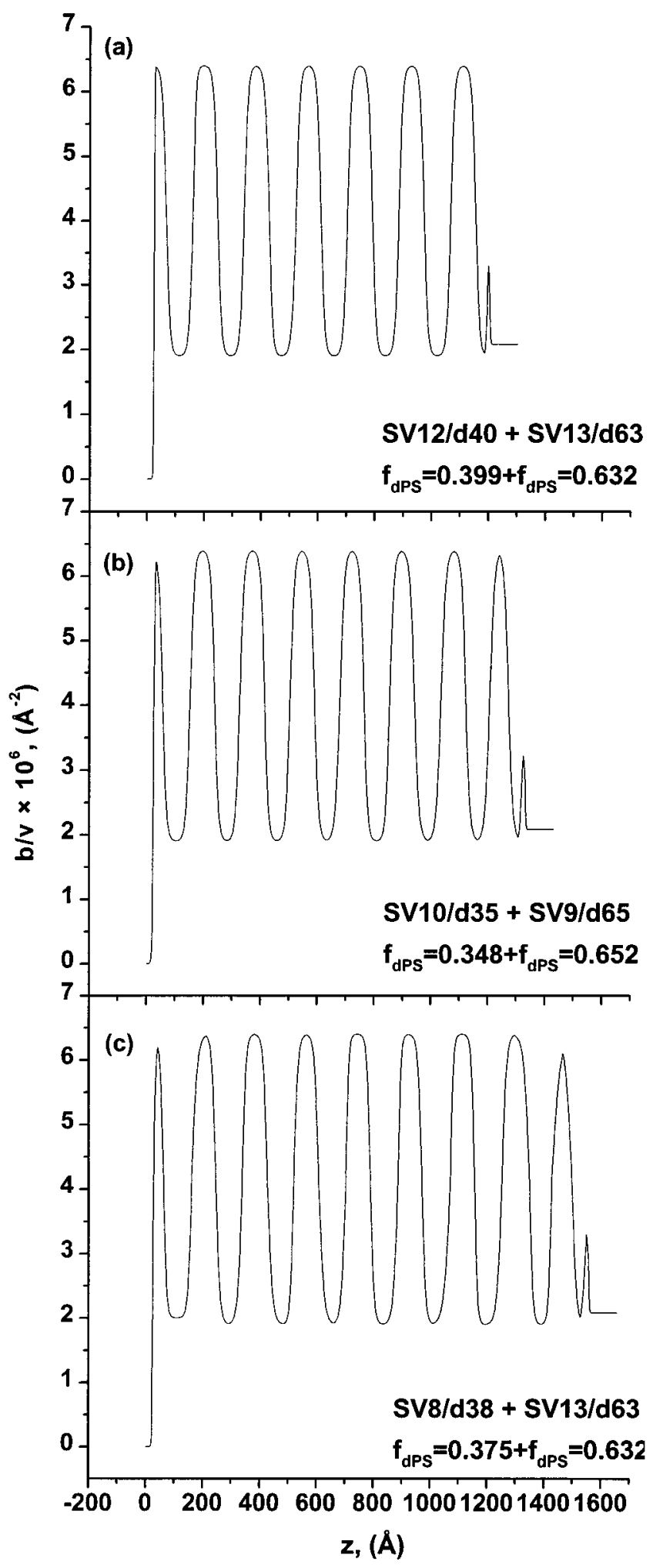

Figure 8. Scattering length density profiles of binary blends of asymmetric diblock copolymers used to calculate the reflectivities in Figure 7.

in parts $a, b$, and $c$ of Figure 7 are $0.233,0.304$, and 0.257 , respectively. Note that $f_{A, \alpha}+f_{A, \beta}$ is nearly a constant $(\approx 1)$ and the degree of polymerization is also nearly constant, $\mathrm{N}_{\alpha} \approx \mathrm{N}_{\beta}(\approx 200)$. Under these conditions, self-consistent field calculations ${ }^{10,13}$ predict that the two blend components are molecularly miscible and form a lamellar blend morphology consistent with our experimental findings. 


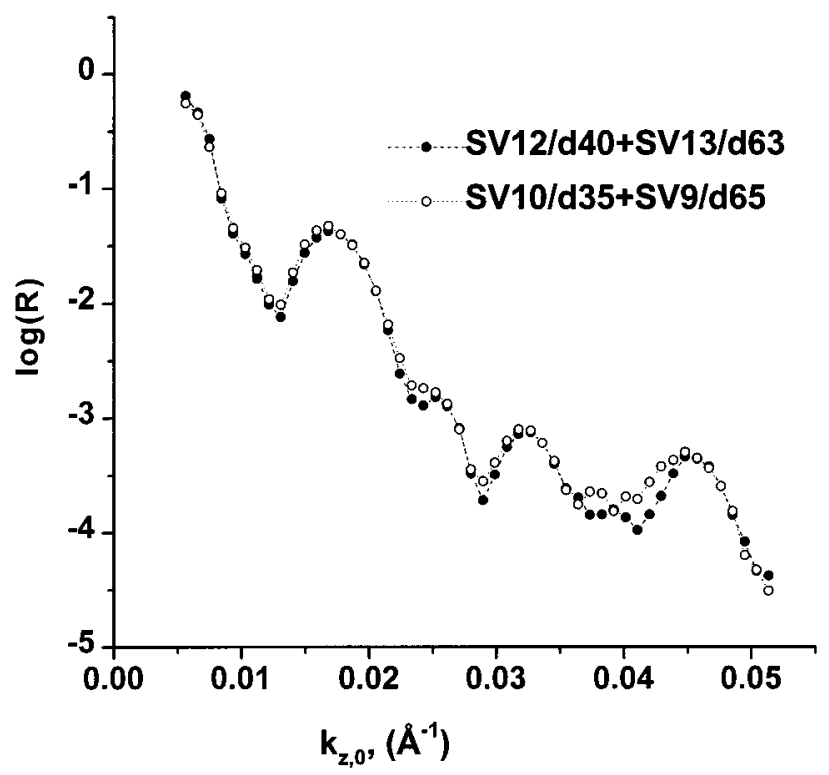

Figure 9. Neutron reflectivity data (open symbols) from binary blends of asymmetric diblock copolymers of dPSPVP. The profiles were obtained from samples with a film thickness of about $450 \AA$. The superposition of the reflectivity profiles suggests a similar morphology (lamellar) in both samples.

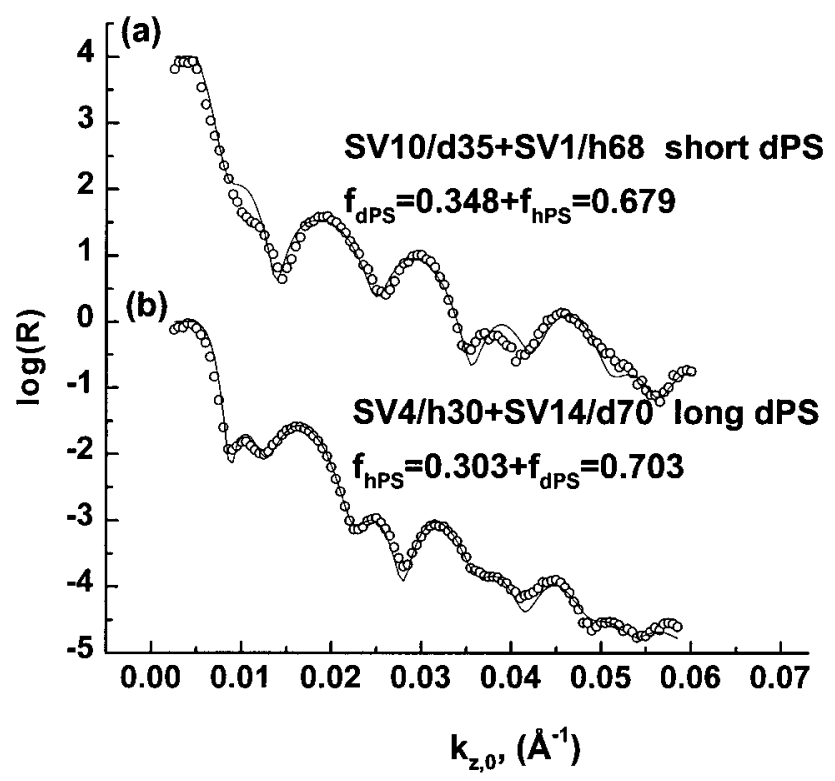

Figure 10. Neutron reflectivity profiles (open symbols) from binary blends of asymmetric diblock copolymers. The profiles are vertically offset by two decades. In a, the short PS block of the diblock is perdeuterated, while in $b$, the long PS block of the diblock is perdeuterated. The solid lines are calculated reflectivities from the SLD profiles in Figure 11.

In the morphology of the multicomponent samples discussed thus far, blending is accompanied by changes in packing constraints at the internal interfaces of the ordered structure. Mixing of short and long chains negates the spontaneous interfacial curvature associated with the individual components. Such rearrangements introduce distortions to the chain configurations, and the structural features can be addressed by preferentially labeling either the short or the long chain (see below).

Figure 10 shows reflectivity profiles from binary blends of asymmetric diblock copolymers with contrast

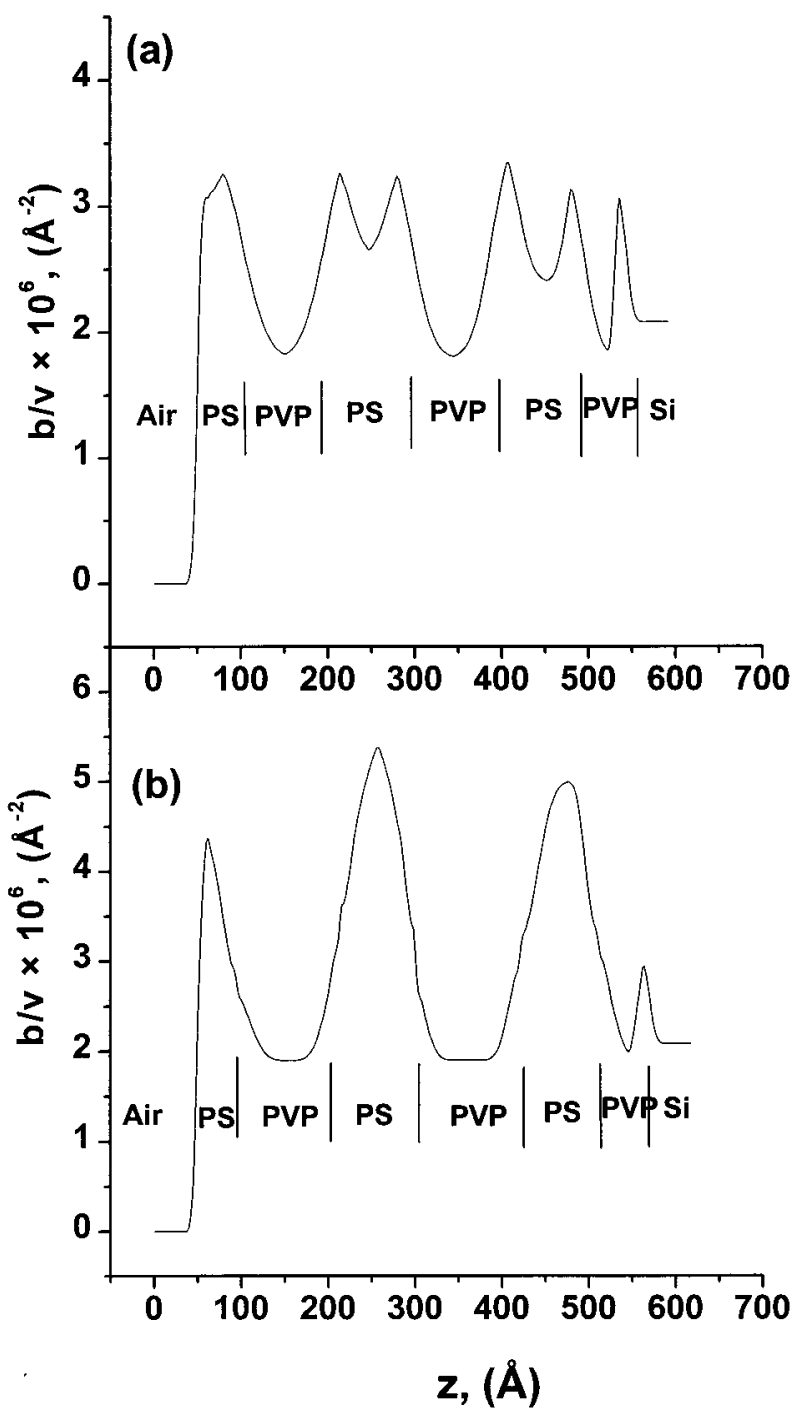

Figure 11. Scattering length density profiles used to calculated the reflectivities in Figure 10.

between the short and long PS blocks. In Figure 10a, the short PS block is perdeuterated, while in Figure 10b, the long PS block is perdeuterated. The total volume fraction of PS in each of the blends is constant $\left(\mathrm{f}_{\mathrm{A}, \alpha}+\right.$ $\mathrm{f}_{\mathrm{A}, \beta} \approx 1$ ). To facilitate neutron reflectivity data analysis, it was desirable to minimize the number of interfaces in the film. Hence, samples with a lower film thickness corresponding to 2.5 bilayers were prepared.

Strong Bragg reflections are not seen in the reflectivity profiles due to a lower film thickness and nonuniform SLD in the PS-rich domains. The degree of compositional asymmetry $\left(\left|f_{A, \alpha}-f_{A, \beta}\right|\right)$ is 0.331 in Figure $10 \mathrm{a}$ and 0.400 in Figure 10b. From the results presented earlier (Figure 7 and Figure 8), a lamellar morphology is expected in these bl ends. The solid lines are reflectivity profiles calculated from the SLD profiles in Figure 11. The agreement between experiment and calculated reflectivity is reasonably good. There is, however, a slight mismatch between the two in the blend sample containing short dPS blocks. The bumps at $k_{z, 0}=0.01$ and $0.038 \AA^{-1}$ are not well captured by the calculated reflectivity profile. Model fitting of the data is complicated by the recurrent changes in curvature in the SLDs (Figure 11). The mismatch is not too serious considering that all the qualitative features of the experimental data are captured in the calculated 
profile. Variations in the SLDs in the PS domains are due to a nonuniform distribution of the hydrogenated and perdeuterated PS chains. Both SLD profiles in Figure 11 indicate segregation of the long dPS chains to the center of the PS domain and the short dPS chains to the edges of the PS domains. These results are qualitatively similar to the results from blends of symmetric diblock copolymers discussed in the previous section. Furthermore, in the SLD profiles of both blends, there appears to be a slight asymmetry in the distribution of the two components close to the substrate interfaces. Deviations from a homogeneous distribution of the two components near these surfaces could be due to the pinning effect of the surfaces. This was observed in a blend of symmetric block copolymers as well (for the sample $\phi_{\mathrm{h} 120}=0.60$ in the previous section). Longer annealing times may be required to overcome such effects. The origin of these effects is not fully understood at present.

The SLD profiles were inverted to volume fraction profiles using the procedures described earlier and compared with SCF calculations. The molecular parameters needed for SCF calculations are provided in Table 1. The portion of the volume fraction profile between $z \approx 150$ and $350 \AA$ was used for purposes of comparison with SCF calculations. The overall volume fraction of PS (hPS + dPS) is 0.481 (Figure 11a) and 0.478 (Figure $11 \mathrm{~b}$ ). The results from the experiment and SCF calculations for two values of the interaction parameter, $\chi=0.10$ and 0.14 , are presented in Figure 12. It is clear from the figure that the SCF calculation predicts a sharper interface between the PS and the PVP domains than the data suggest. The SCF profiles also illustrate a greater sensitivity to $\chi$ at lower $\mathrm{N}$ (cf. Figure 3). It appears that a higher value of $\chi$ is necessary to fit the data from asymmetric blends than the bulk value.

The higher value of $\chi$ needed to model the lower molecular weight data suggests an increased degree of order in the film. This could be due to enhancement of ordering arising from the presence of surfaces near the order-disorder temperature (ODT). The copolymers employed in the study of asymmetric blends $(N \approx 200)$ have order-disorder transitions in the vicinity of 200 ${ }^{\circ} \mathrm{C},{ }^{14}$ and the experiments in this study were conducted at $165-170{ }^{\circ} \mathrm{C}$, which is fairly close to the ODT. However, the presence of surface-induced orientation implies that the system is further from the ODT than the bulk values would suggest. Regardless, SCF calculations from the two values of $\chi(0.10$ and 0.14$)$ yield reasonable agreement to the data within the uncertainties in the experiment.

In Table 2, we provide domain sizes and interfacial widths (interface width is defined as the inverse slope of the tangent to the $\mathrm{A}-\mathrm{B}$ composition profile at the interface midpoint ${ }^{19}$ ) obtained from experiment and SCF calculations. The values from experiment for domain periods and the interfacial widths are averaged over all the bilayers in the film. The values from SCF are obtained using $1 / \rho_{0}=156 \AA^{3}$ and $a_{A}=a_{B}=6.7 \AA$. The calculated domain sizes for $\chi=0.14$ agree well with experiment, with one exception, which we will discuss at the end of this section. The SCF calculation evaluates the interfacial width assuming that the interface is stationary, However, in experiments, capillary wave fluctuations produce an effective broadening of the interface. ${ }^{25}$ Reference 26 cal culates a correction for this

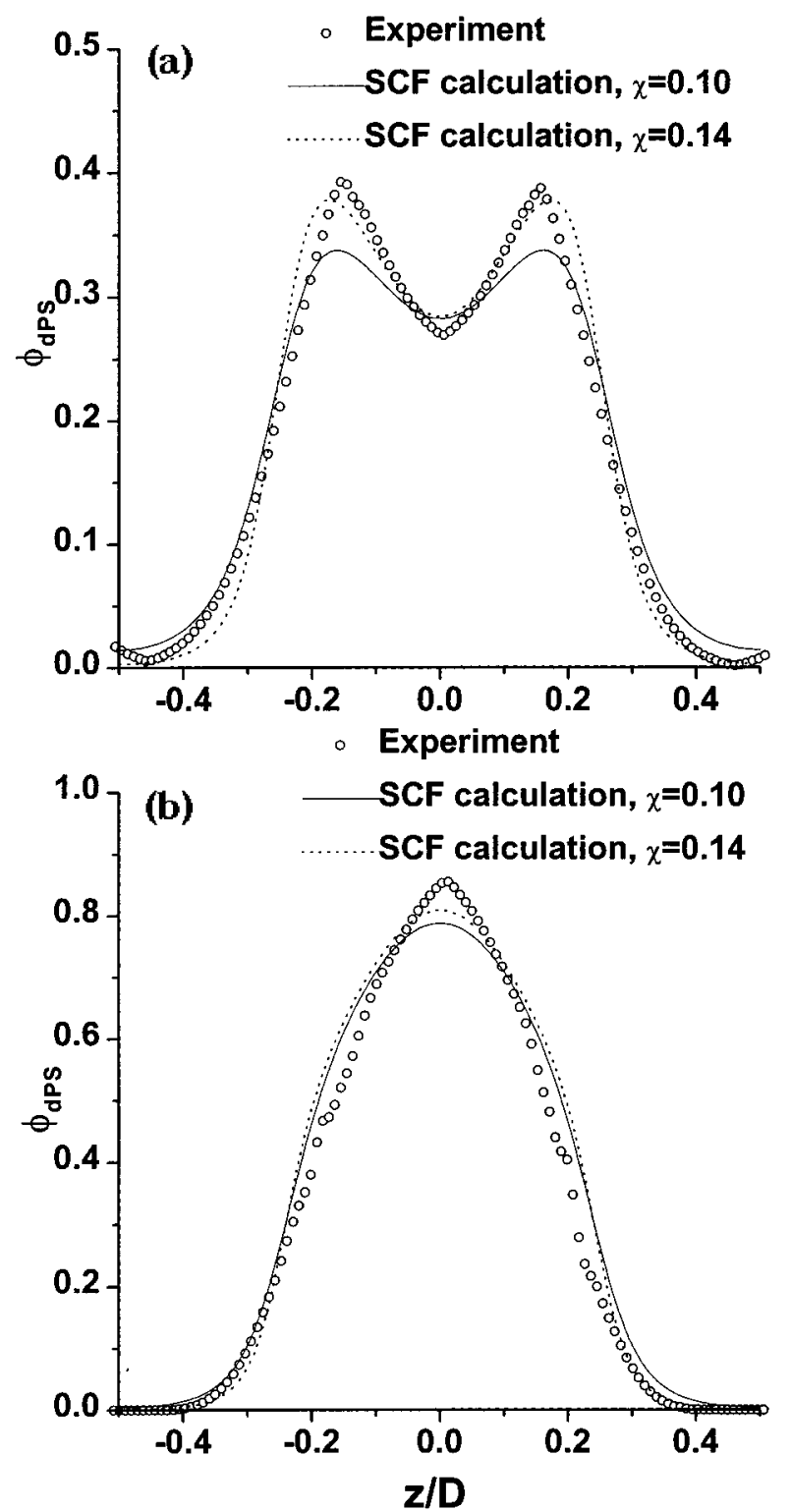

Figure 12. Volume fraction of long dPS chains (a) and short dPS chains (b) from experiment (open symbols) and SCF calculations.

effect. The mean-square displacement of the interface from its average position is given by

$$
\left\langle(\Delta z)^{2}\right\rangle=\frac{k_{B} T}{2 \pi \gamma_{A B}} \ln \left(\frac{\lambda_{\max }}{\lambda_{\min }}\right)
$$

where $\gamma_{A B}$ is the interfacial tension between the two polymers and $\lambda_{\max }$ and $\lambda_{\min }$ are the maximum and minimum wavel ength of fluctuations. They are taken to be equal to the domain period and the intrinsic interfacial width, respectively. ${ }^{26} \gamma_{A B}$ is estimated using the strong segregation theory of Helfand and co-workers: ${ }^{27}$

$$
\gamma_{\mathrm{AB}}=\mathrm{a} \rho_{0} \mathrm{k}_{\mathrm{B}} \mathrm{T}\left(\frac{\chi}{6}\right)^{1 / 2}
$$

The statistical segment lengths of PS and PVP are assumed to be equal $(=6.7 \AA)$, the density of the copolymer, $\rho_{0}$ is taken to be $1 / \rho_{0}=156 \AA^{3}$, yielding the values of $\gamma_{A B}$ at $170{ }^{\circ} \mathrm{C}$ to be 3.35 and $3.96 \mathrm{~mJ} / \mathrm{m}^{2}$ for $\chi$ $=0.10$ and 0.14 , respectively. The fluctuation correction 
Table 2. Comparison of Domain and Interfacial Widths Obtained from Neutron Reflection Experiments, from SCF Calculations, and with Corrections Due to Interface Fluctuations ${ }^{a}$

\begin{tabular}{|c|c|c|c|c|c|c|c|c|}
\hline \multirow[b]{3}{*}{ blends } & \multicolumn{3}{|c|}{ domain width, $\AA$} & \multicolumn{5}{|c|}{ interface width, $\AA$} \\
\hline & \multirow[b]{2}{*}{ expt } & \multicolumn{2}{|c|}{ theory } & \multirow[b]{2}{*}{ expt } & \multicolumn{2}{|c|}{ uncorrected } & \multicolumn{2}{|c|}{ corrected } \\
\hline & & $\chi=0.10$ & $\chi=0.14$ & & $\chi=0.10$ & $\chi=0.14$ & $\chi=0.10$ & $\chi=0.14$ \\
\hline d120/d60 & 364 & 388 & 415 & $40.0^{b}$ & 21.9 & 18.0 & 31.6 & 28.3 \\
\hline $\mathrm{SV} 12 / \mathrm{d} 40+\mathrm{SV} 13 / \mathrm{d} 63$ & 181 & 164 & 178 & 26.2 & 26.6 & 20.6 & 32.2 & 27.5 \\
\hline SV10/d35 + SV9/d65 & 176 & 162 & 175 & 29.7 & 27.4 & 21.0 & 32.8 & 27.7 \\
\hline SV8/d38 + SV13/d63 & 180 & 160 & 174 & 29.4 & 27.1 & 20.8 & 32.5 & 27.6 \\
\hline
\end{tabular}

a Note that $\chi$ and $N$ are calculated assuming $1 / \rho_{0}=156 \AA^{3} .{ }^{b}$ Value for this sample is an artifact associated with $N R$ fitting (see text).

in the interfacial width is given by $\left(2 \pi\left\langle\Delta z^{2}\right\rangle\right)^{1 / 2}$ and is added in quadrature to the intrinsic interfacial width (obtained from SCF).

The structural features in the d120/d60 sample do not agree well with the SCF calculations (see Table 2). The domain size is significantly smaller than predicted, and the interfacial width of $40 \AA$ is larger than expected. The same copolymer (i.e., d60 and d120) in another set of experiments ${ }^{28}$ where the samples were annealed at $185^{\circ} \mathrm{C}$ yiel ded interfacial widths between 25 and $30 \AA$, consistent with all other PS-PVP copolymer samples employed in this study. We believe that this is the correct value for PS/PVP interfacial width and this value does not vary significantly in the range of molecular weights or blend ratios examined. The $40-\AA$ interfacial width value for the d120/d60 sample is an artifact associated with fitting of the neutron reflectivity profile from the d120/d60 sample. This sample had holes on the free surface that could not be exactly accounted for in the SLD profile (in the domain adjacent to the free surface). This result, however, was used in comparing the SCF calculations with the h120/d60 blends and contributes to the discrepancy between the two in the interfacial zone. Clearly, the agreement between the experimental profiles and SCF calculation (in Figure 3 the interfacial region) would be better if this discrepancy was accounted for. It is difficult to draw definite conclusions from the data with regard to the value of $\chi$ that would fit the composition profiles of symmetric diblock copolymer blends. For the blends of asymmetric diblock copolymers, however, the corrected interfacial widths and the domain periods obtained for $\chi=0.14$ agree well with experiment. A higher value of $\chi$ (with respect to bulk) as deduced from SCF calculations points to surface-enhanced order in thin films.

\section{Conclusions}

We have obtained the short- and long-chain distribution profiles in binary blends of symmetric and asymmetric diblock copolymers. The blend composition profiles show nonuniformity in the distribution of the chains with the longer chains segregating to the domain centers and shorter chains more localized near the interfaces. Blending diblock copolymers with opposite signs of their spontaneous curvature resulted in molecularly miscible planar-lamellar morphology even though the pure component morphologies were nonlamellar. This was accompanied by stretching of the chains so that the domain period of the blends was slightly ( $5 \%)$ larger than the domain period of either of the pure components. In addition, under conditions close to the bulk order-disorder temperature, ordering in the thin films appeared to be enhanced due to the presence of the film surfaces. After accounting for fluctuations of the interfaces, self-consistent field cal- culations were in quantitative agreement with the asymmetric blends data. This work reaffirms the capability and reliability of self-consistent field calculations to accurately describe multicomponent block copolymers and other systems not readily accessible for experimentation.

Acknowledgment. Support for this research was provided by the National Science Foundation (Grant DMR-9405101) and the Center for Interfacial Engineering, a National Science Foundation Engineering $\mathrm{Re}$ search Center at the University of Minnesota. Nagraj Koneripalli acknowledges financial support from the Graduate School at the University of Minnesota through a Doctoral Dissertation Fellowship.

\section{References and Notes}

(1) Hashimoto, T.; Yamasaki, K.; Koizumi, S.; Hasegawa, H. Macromol ecules 1993, 26, 2895.

(2) Hadziioannou, G.; Skoulios, A. Macromolecules 1982, 15, 267.

(3) Koizumi, S.; Hasegawa, H.; Hashimoto, T. Macromolecules 1994, 27, 4371.

(4) Vilesov, A. D.; Floudas, G.; Pakula, T.; Melenevskaya, E. Y.; Birshtein, T. M.; Lyatskaya, Y. V. Macromol. Chem. Phys. 1994, 195, 2317.

(5) Hashimoto, T.; Koizumi, S.; Hasegawa, H. Macromolecules 1994, 27, 1562.

(6) Spontak, R. J . Macromolecules 1996, 29, 4494.

(7) Mayes, A. M.; Russell, T. P.; Deline, V. R.; Satija, S. K.; Majkrzak, C. F. Macromolecules 1994, 27, 7447.

(8) Zhao, J .; Majumdar, B.; Schulz, M. F.; Bates, F. S.; Almdal, K.; Mortensen, K.; Hajduk, D. A.; Gruner, S. M. Macromolecules 1996, 29, 1204.

(9) Schulz, M. F.; Bates, F. S.; Almdal, K.; Mortensen, K. Phys. Rev. Lett. 1994, 73, 86.

(10) Matsen M. W.; Bates, F. S. Macromolecules 1995, 28, 7298.

(11) Spontak, R. J . Macromolecules 1994, 27, 6363; Zhulina, E. B.; Birshtein, T. M. Polymer 1991, 32, 1299; Dan, N.; Safran, S. A. Macromolecules 1994, 27, 5766.

(12) Matsen, M. W. J . Chem. Phys. 1995, 103, 3268

(13) Shi, A.-C.; Noolandi, J .; H offman, H. Macromolecules 1994, 27,6661 .

(14) Schulz, M. F.; Khandpur, A. K.; Bates, F. S.; Almdal, K.; Mortensen, K.; Hajduk, D. A.; Gruner, S. M. Macromol ecules 1996, 29, 2857.

(15) In test samples of PS-PVP films, a refractive index of 1.58 for $\lambda=6328 \AA$ yielded the best agreement in film thickness measured by ellipsometry and X-ray reflectivity.

(16) Koneripalli, N.; Levicky, R.; Bates, F. S.; Ankner, J . F.; Kaiser, H.; Satija, S. K. Langmuir 1996, 12, 6681 and references therein.

(17) Ankner, J . F.; Majkrzak, C. F.; Satija, S. K. J . Res. Nat. Inst. Stand. Technol. 1993, 98, 47.

(18) Kaiser, H.; Hamacher, K.; Kulasekere, R.; Lee, W.-T.; Ankner, J F.; DeF acio, B.; Miceli, P.; Worcester, D. L. Inverse Optics III, SPIE Conf. Proc. 1994, 2241, 78.

(19) Helfand, E. J . Chem. Phys. 1975, 62, 999.

(20) Hong, K. M.; Noolandi, J . Macromolecules 1981, 14, 727.

(21) Matsen, M. W. Phys. Rev. Lett. 1995, 74, 4225; Matsen, M. W. Macromolecules 1995, 28, 5765 . 
3508 Koneripalli et al.

(22) O'Malley et al. Polym. Prepr. 1969, 10, 796; Shull, K. R.; Kramer, E. J .; Hadziioannou, G.; Tang, W. Macromolecules 1990, 23, 4780.

(23) Samples were rapidly cooled from $170^{\circ} \mathrm{C}$ to room temperature through the copolymer glass transition temperature $(\sim 100$ $\left.{ }^{\circ} \mathrm{C}\right)$. Although the measurements were performed at room temperature, the frozen sample morphology is more repre sentative of the annealing conditions, i.e., $170^{\circ} \mathrm{C}$.

(24) The $M_{n}$ values of the two diblock copolymers SV12/d40 and SV13/d63 are similar (viz. 21.8 and 21.7, respectively; see Table 1) so that their domain periods are expected to be nearly the same.
Macromolecules, Vol. 31, No. 11, 1998

(25) Sferrazza, M.; Xiao, C.; J ones, R. A. L.; Bucknall, D. G.; Penfold, J. Phys. Rev. Lett. 1997, 78, 3693.

(26) Shull, K. R.; Mayes, A. M.; Russell, T. P. Macromolecules 1993, 26, 3929. Semonov, A. N. Macromolecules 1993, 26, 6617.

(27) Helfand, E.; Sapse, A. M. J . Chem. Phys. 1975, 62, 1327.

(28) Levicky, R.; Koneripalli, N.; Tirrell, M.; Satija, S. K.; Gallagher, P. D.; Ankner, J .; Kulasekere, R.; Kaiser, H. Macromolecules, in press.

MA971229A 